\title{
PUBLISHER CORRECTION
}

\section{Publisher Correction to: Motivations and expectations for using cannabis products to treat pain in humans and dogs: a mixed methods study}

\author{
Jean E. Wallace ${ }^{1 *}$, Lori R. Kogan², Eloise C. J. Carr ${ }^{3}$ and Peter W. Hellyer ${ }^{2}$
}

Publisher Correction to: J Cannabis Res 2, 36 (2020)

https://doi.org/10.1186/s42238-020-00045-x

Following publication of the original article (Wallace et al. 2020), the authors reported that the article had published with errors in some of the tables; data had been erroneously omitted from Tables 1, 2 and 6, and these tables had been incorrectly formatted.

The original article has since been updated to correct the tables.

Furthermore, please find the (corrected) tables in this correction for reference.

The publisher apologizes for this error and any inconvenience caused.

\section{Author details}

'Department of Sociology, University of Calgary, 2500 University Drive NW, Calgary, AB T2N 1 N4, Canada. ${ }^{2}$ College of Veterinary Medicine and Biomedical Sciences, Colorado State University, Fort Collins, CO 80523, USA.

${ }^{3}$ Faculty of Nursing, University of Calgary, 2500 University Dr NW, Calgary, AB T2N $1 \mathrm{~N} 4$, Canada.

Published online: 20 November 2020

\section{Reference}

Wallace JE, Kogan LR, Carr ECJ, et al. Motivations and expectations for using cannabis products to treat pain in humans and dogs: a mixed methods study. J Cannabis Res. 2020;2:36. https://doi.org/10.1186/s42238-020-00045-x.

The original article can be found online at https://doi.org/10.1186/s42238 020-00045.

* Correspondence: jwallace@ucalgary.ca

1 Department of Sociology, University of Calgary, 2500 University Drive NW, Calgary, AB T2N 1 N4, Canada

Full list of author information is available at the end of the article

Ready to submit your research? Choose BMC and benefit from:

- fast, convenient online submission

- thorough peer review by experienced researchers in your field

- rapid publication on acceptance

- support for research data, including large and complex data types

- gold Open Access which fosters wider collaboration and increased citations

- maximum visibility for your research: over $100 \mathrm{M}$ website views per year

At BMC, research is always in progress.

Learn more biomedcentral.com/submissions 
Table 1 Comparisons by Causes and Length of Time with Chronic Pain for Human Patients versus Dog Patients

\begin{tabular}{|c|c|c|c|}
\hline \multirow{2}{*}{\multicolumn{2}{|c|}{$\begin{array}{l}\text { Human Patients }(N=313) \\
\text { Primary Cause of Chronic Pain }\end{array}$}} & \multirow{2}{*}{\multicolumn{2}{|c|}{$\begin{array}{l}\text { Dog Patients }(N=204) \\
\text { Primary Cause of Chronic Pain }\end{array}$}} \\
\hline & & & \\
\hline Chronic Back Pain & $48 \%(149)$ & Chronic Back Pain & $22 \%(45)$ \\
\hline Degenerative Joint Disease & $17 \%(54)$ & Degenerative Joint Disease & $45 \%(91)$ \\
\hline Mouth Pain or Headache & $17 \%(52)$ & Mouth Pain from Dental Disease & $7 \%(14)$ \\
\hline Other & $18 \%(58)$ & Other & $26 \%(54)$ \\
\hline \multicolumn{4}{|l|}{$\times 2(3, N=571)=67.22, p<.001$} \\
\hline Length of Time with Chronic Pain & & Length of Time with Chronic Pain & \\
\hline$<1$ year & $18 \%(55)$ & $<1$ year & $36 \%(74)$ \\
\hline $1-5$ years & $47 \%(146)$ & $1-3$ years & $55 \%(112)$ \\
\hline$>5$ years & $35 \%(108)$ & $>3$ years & $9 \%(18)$ \\
\hline$X 2(2, N=513)=52.26, p<.001$ & & & \\
\hline
\end{tabular}

Table 2 Descriptive Information for Cannabis Products Used and Comparison for How Cannabis Products are Obtained for Human Patients and Dog Patients

\begin{tabular}{|c|c|c|c|}
\hline \multirow{2}{*}{$\begin{array}{l}\text { Human Patients }(N=313) \\
\text { Type of Cannabis Product Used }\end{array}$} & & \multicolumn{2}{|l|}{ Dog Patients $(N=204)$} \\
\hline & & Types of Cannabis Product Used ${ }^{a}$ & \\
\hline Marijuana/Cannabis (THC > 0.3\%) & $76 \%(237)$ & Marijuana/Cannabis (THC > 0.3\%) & $26 \%(53)$ \\
\hline Hemp Isolate $(\mathrm{THC}<0.3 \%)$ & $49 \%(152)$ & Hemp Isolate $(\mathrm{THC}<0.3 \%)$ & $44 \%(89)$ \\
\hline CBD/Hemp Broad or Full Spectrum & $36 \%(113)$ & CBD/Hemp Broad or Full Spectrum & $42 \%(88)$ \\
\hline Not Sure & $3 \%(9)$ & Not Sure & $11 \%(22)$ \\
\hline Most Frequent Way of Obtaining Cannabis & & Most Frequent Way of Obtaining $\mathrm{Ca}$ & \\
\hline Given by Friend or Family & $33 \%(102)$ & Given by Friend or Family & $11 \%(22)$ \\
\hline Dispensary or Store & $30 \%(92)$ & Dispensary or Store & $25 \%(50)$ \\
\hline Natural/Health Store/Service & $12 \%(40)$ & Natural/Health Store/Service & $25 \%(50)$ \\
\hline Online Source & $12 \%(36)$ & Online Source & $34 \%(68)$ \\
\hline Other & $13 \%(43)$ & Other & $5 \%(14)$ \\
\hline$\times 2(4, N=517)=69.87, p<.001$ & & & \\
\hline
\end{tabular}

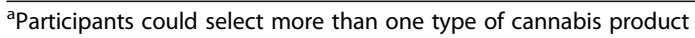

Table 6 Percentages of Respondents Who Felt their Expectations were Met by Cannabis Products Used and Obtained

\begin{tabular}{|c|c|c|c|}
\hline \multirow{2}{*}{$\begin{array}{l}\text { Human Patients }(N=313) \\
\text { Type of Cannabis Product Used }\end{array}$} & & \multicolumn{2}{|l|}{ Dog Patients $(N=204)$} \\
\hline & & Types of Cannabis Product Used $^{\mathrm{a}}$ & \\
\hline Marijuana/Cannabis (THC > 0.3\%) & $88 \%(199)$ & Marijuana/Cannabis (THC > 0.3\%) & $94 \%(45)$ \\
\hline Hemp Isolate (THC < 0.3\%) & $83 \%(120)$ & Hemp Isolate (THC < 0.3\%) & $76 \%(68)$ \\
\hline CBD/Hemp Broad or Full Spectrum & $90 \%(99)$ & CBD/Hemp Broad or Full Spectrum & $87 \%(75)$ \\
\hline Not Sure & $67 \%(6)$ & Not Sure & $68 \%(15)$ \\
\hline Most Frequent Way of Obtaining Cannabis & & Most Frequent Way of Obtaining $\mathrm{Ca}$ & \\
\hline Given by Friend or Family & $86 \%(88)$ & Given by Friend or Family & $77 \%(17)$ \\
\hline Dispensary or Store & $85 \%(78)$ & Dispensary or Store & $84 \%(42)$ \\
\hline Natural/Health Store/Service & $90 \%(36)$ & Natural/Health Store/Service & $84 \%(42)$ \\
\hline Online Source & $78 \%(36)$ & Online Source & $82 \%(56)$ \\
\hline Other & $80 \%(20)$ & Other & $63 \%(9)$ \\
\hline
\end{tabular}

Participants could select more than one type of cannabis product 